\title{
A systematic review assessing the potential for release of vector species from competition following insecticide-based population suppression of Anopheles species in Africa
}

\author{
Alima Qureshi and John B. Connolly ${ }^{*}$ (D)
}

\begin{abstract}
Background: While insecticide-based vector control can effectively target vector species in areas of high malaria endemicity, such as Anopheles gambiae in Africa, residual disease transmission can occur. Understanding the potential role of competitive displacement between vector species could inform both current insecticide-based vector control programmes and the development of future complementary interventions.

Methods: A systematic review was conducted to identify published studies of insecticide-based vector control of Anopheles species in Africa that reported indices for absolute densities of vector species. After screening against inclusion, exclusion and risk of bias criteria, studies were assigned to three categories based on whether they showed population density changes involving decreases in two or more vector species (D), increases in two or more vector species (I), or increases in one vector species concomitant with decreases in another vector species (ID). Category ID studies could thus provide evidence consistent with the release of vector species from competition following the insecticide-based population suppression of Anopheles species.

Results: Of 5569 papers identified in searches, 30 were selected for quantitative and qualitative analysis. Nineteen studies were assigned to category D and one to category I. Ten studies categorised as ID provided evidence ranging from weak to persuasive that release from competition could have contributed to changes in species composition. Category ID showed no statistical differences from category $D$ for reductions in malaria transmission and levels of insecticide resistance, but did so for insecticide type, pyrethroids being associated with category ID. A qualitative assessment identified five studies that provided the most convincing evidence that release from competition could have contributed to changes in species composition.

Conclusions: This review identified evidence that insecticide-based reductions in the density of Anopheles species in Africa could facilitate the release of other vector species from competition. While it remains uncertain whether this evidence is representative of most entomological sequelae of insecticide-based vector control in the field, five studies provided persuasive evidence that insecticide use could lead, at least under some circumstances, to competitive release of non-targeted vector species. These results should inform current and future integrated vector management approaches to malaria control.
\end{abstract}

Keywords: Anopheles, Vector control, Competitive release, Niche replacement, Insecticide

*Correspondence: john.connolly12@imperial.ac.uk

Department of Life Sciences, Imperial College London, Silwood Park

Campus, Ascot SL5 7PY, UK

(c) The Author(s) 2021. Open Access This article is licensed under a Creative Commons Attribution 4.0 International License, which permits use, sharing, adaptation, distribution and reproduction in any medium or format, as long as you give appropriate credit to the original author(s) and the source, provide a link to the Creative Commons licence, and indicate if changes were made. The images or other third party material in this article are included in the article's Creative Commons licence, unless indicated otherwise in a credit line to the material. If material is not included in the article's Creative Commons licence and your intended use is not permitted by statutory regulation or exceeds the permitted use, you will need to obtain permission directly from the copyright holder. To view a copy of this licence, visit http://creativecommons.org/licenses/by/4.0/. The Creative Commons Public Domain Dedication waiver (http://creativeco mmons.org/publicdomain/zero/1.0/) applies to the data made available in this article, unless otherwise stated in a credit line to the data. 


\section{Background}

An ecological niche can be considered as the habitat supplying the resources that are required for the survival and reproduction of a species, or the roles and interactions that one species has within a community of other species [1]. Competitive reduction reflects situations where the density of the population of one species decreases because of competitive interactions, directly or indirectly, with another. Competitive displacement is that more extreme situation where competitive interactions of one species with another causes its local elimination. Competitive interactions between sympatric vector species could thus potentially play a role in continued disease transmission [1]. Aquatic habitats for mosquito larval development are regarded as having the most impact on adult population densities [2,3]. Here, competition can occur via (i) exploitation, where individuals compete for limited resources, (ii) interference, where individuals obstruct the development of others, (iii) apparent competition, where there are differential effects of a predator or parasite on co-occurring species, or (iv) oviposition deterrence, where egg-laying by one species is avoided where a competitor species is present [1]. Therefore, differential effects of vector control measures on sympatric vector species could facilitate the release of co-located vector species from competitive displacement or reduction by the targeted species, sometimes termed 'release from competition' or 'competitive release', leading to 'niche replacement' or 'niche expansion' of other vector species, potentially facilitating residual or increased transmission of vector-borne infections.

Insecticide-based vector control interventions, such as long-lasting insecticide-treated bednets (LLINs) and indoor residual spraying (IRS), are known to more effectively target vector species that are endophilic, anthropophilic and nocturnal. In Africa, the dominant malaria vectors are Anopheles gambiae sensu stricto (s.s.), Anopheles coluzzii, Anopheles arabiensis and Anopheles funestus [4]. Although An. funestus is a complex consisting of 13 species within Africa, of these, only the species $A n$. funestus is thought to play a role in malaria transmission [5]. While the above four named species typically engage in nocturnal blood-feeding, An. funestus and An. arabiensis can also exhibit crepuscular and in some instances diurnal behaviour [6, 7]. Anopheles gambiae s.s. and An. coluzzii are endophilic and anthropophilic, in contrast to their sibling species An. arabiensis, which displays exophilic, zoophilic behaviour. Thus, sufficient numbers of less efficiently targeted vector species could possibly remain in the area of vector control and contribute to residual, or even resurgent, transmission of malaria.

Differences in characteristics of individual Anopheles species could further lead to a range of sensitivities of sympatric vectors to insecticides, impacting on behavioural responses seen post-intervention. Some species are known to be behaviourally resilient, exhibiting plasticity, rather than showing evidence of altered innate preferences and subsequent 'resistance' to intervention [8]. These differences could potentially aid and further the strength of any competitive interaction.

Moreover, An. gambiae s.s. and An. arabiensis are sibling species known to co-occur within aquatic habitats $[9,10]$. Mesocosm experiments show that competition affects the development rate of each species in the opposite way. Development time for An. arabiensis increases in the presence of An. gambiae s.s. and decreases for An. gambiae s.s. in the presence of An. arabiensis [11]. In the laboratory, An. arabiensis shows reduced survival when reared with An. gambiae s.s. compared to when reared alone [12]. When employing targeted vector control measures, it is thus possible to envisage that reduced densities of An. gambiae s.s. could facilitate competitive release of An. arabiensis. However, some caution should be exercised in interpreting whether mesocosm and laboratory-based data are representative of field settings. Indeed, sampling of Anopheles larvae from aquatic habitats can yield highly heterogeneous densities [13], making field studies exploring competitive mechanisms potentially challenging.

Nonetheless, understanding the dynamics of changes in vector species composition and any competitive reduction or displacement could be important for the effective use of current insecticide-based vector control programmes and could inform the development of novel interventions [14]. While evidence for protective effects of insecticide-based methods of vector control is convincing [15], between 2014 and 2019 previous global gains in malaria control decelerated, particularly in Africa, where the burden of malaria is highest [16]. Although there is extensive literature on studies of competitive interactions between mosquito vector species outside of Africa [1, 17-19], the synthesis of studies within Africa investigating the impacts of insecticide-based vector control on competitive interactions between species of Anopheles and other vector species remains more limited [14, 20]. Moreover, while numerous studies from Africa have demonstrated that use of insecticides can result in substantial population suppression of dominant malaria vectors, such as An. gambiae s.s. or An. funestus, many have typically reported changes in the relative proportions, rather than absolute densities, of targeted species to nontarget ones [21-24], making it impossible to differentiate reliably between established preferential effects of insecticides on target versus non-target species and any genuine increases in the densities of non-target species that might indicate true competitive release. 
Thus, the extent of evidence showing decreases in the densities of targeted vector species and concurrent increases in the density or densities of other species remains unclear. Restriction of a synthesis of the impacts of insecticide-based vector control on competitive interactions to African studies would involve analysis of a more discrete set of vector species than would be the case for a global investigation. This could, therefore, elicit a more precise evaluation of the entomological impacts of insecticide-based malaria vector control programmes in Africa. The aim of this systematic review was therefore to critically assess published research to determine whether insecticide-based vector control interventions in Africa targeting Anopheles mosquitoes could potentially facilitate the competitive release of other vector species.

\section{Methods}

\section{Search strategy}

A review protocol was developed in accordance with the Preferred Reporting Items for Systematic Reviews and Meta-Analyses [25] guidelines and registered with the PROSPERO international prospective register of systematic reviews (record ID CRD42020194304) [26, 27]. The systematic literature review was undertaken from June to September 2020, using PubMed, Web of Science and Science Direct. Each was searched the from inception of each search engine until September 2020. Search terms used included 'intervention', 'insecticide', 'insecticide residual spraying,' 'long lasting insecticidal nets', 'anopheles', 'vector,' 'changes' and 'resurgence', the use of which was based on search strings created from three categories: exposure/treatment, subject and outcome. Boolean operators 'OR', 'AND', and 'NOT' were used to narrow or broaden results. Reference lists of selected articles were also searched. EndNote and Microsoft Excel were used as reference manager software.

\section{Eligibility criteria}

Studies identified were screened against eligibility criteria in specific stages to provide clarity on the causes of exclusion. The first stage was undertaken by AQ and involved screening of title and abstract for the following inclusion criteria for studies: within Africa; species of Anopheles mosquitoes included as a mosquito of interest; insecticide-based intervention tested; data contained either before and after, or with and without, intervention arms (Table 1). In the second stage, AQ and JBC conducted full-text reviews of all studies based on the previous inclusion criteria plus the following: inclusion of at least two species of mosquito species, with at least one being from the Anopheles genus; inclusion of Anopheles and other species density outcome data for both before and after or with and without intervention implementation, with accepted density outcome data and trial design criteria, as well as inclusion and exclusion criteria as described in Table 1.

\section{Risk of bias}

The final stage of eligibility assessment required studies to undergo bias assessment through the robvis ROB1 tool, and subsequent exclusion of studies that were found to be at high risk of bias. Bias and validity assessments were undertaken through a set of outlined bias risks, for each individual study satisfying all inclusion criteria (see Additional file 1: Table S1).

\section{Data extraction}

From studies eligible for quantitative and qualitative assessments, AQ extracted data relating to author, year, country, intervention type, insecticide used, vectors involved, replicates for each treatment, absolute densities of mosquito populations and corresponding units of measurement, proportional densities of mosquito populations and corresponding units of measurement, rates of malaria transmission and corresponding units of measurement, bias risk, confounding factors, and any other relevant details or comments.

The following formula was used to calculate the percentage relative reduction in absolute densities of mosquito populations, where 'first value' represents data before or without intervention, and 'second value' represents data after or with intervention:

$$
\begin{aligned}
& \text { Percentage relative reduction }= \\
& \quad 100 *(100-\text { first value/second value })
\end{aligned}
$$

Use of percentage relative reduction meant that any increases in density or transmission were noted as negative numbers, and any decreases were noted as positive numbers (see Additional file 2).

\section{Categorisation of studies}

Following risk of bias assessments, all remaining studies were subsequently assigned to density categories based on reported density changes in vector species:

- Category D-two or more vector species decreased in absolute densities.

- Category I-two or more vector species increased in absolute densities.

- Category ID-one or more vector species decreased in absolute densities, concomitant with increased density in one or more other vector species.

Individual studies were further stratified by data on location, species, date, trap type, and trap placement 


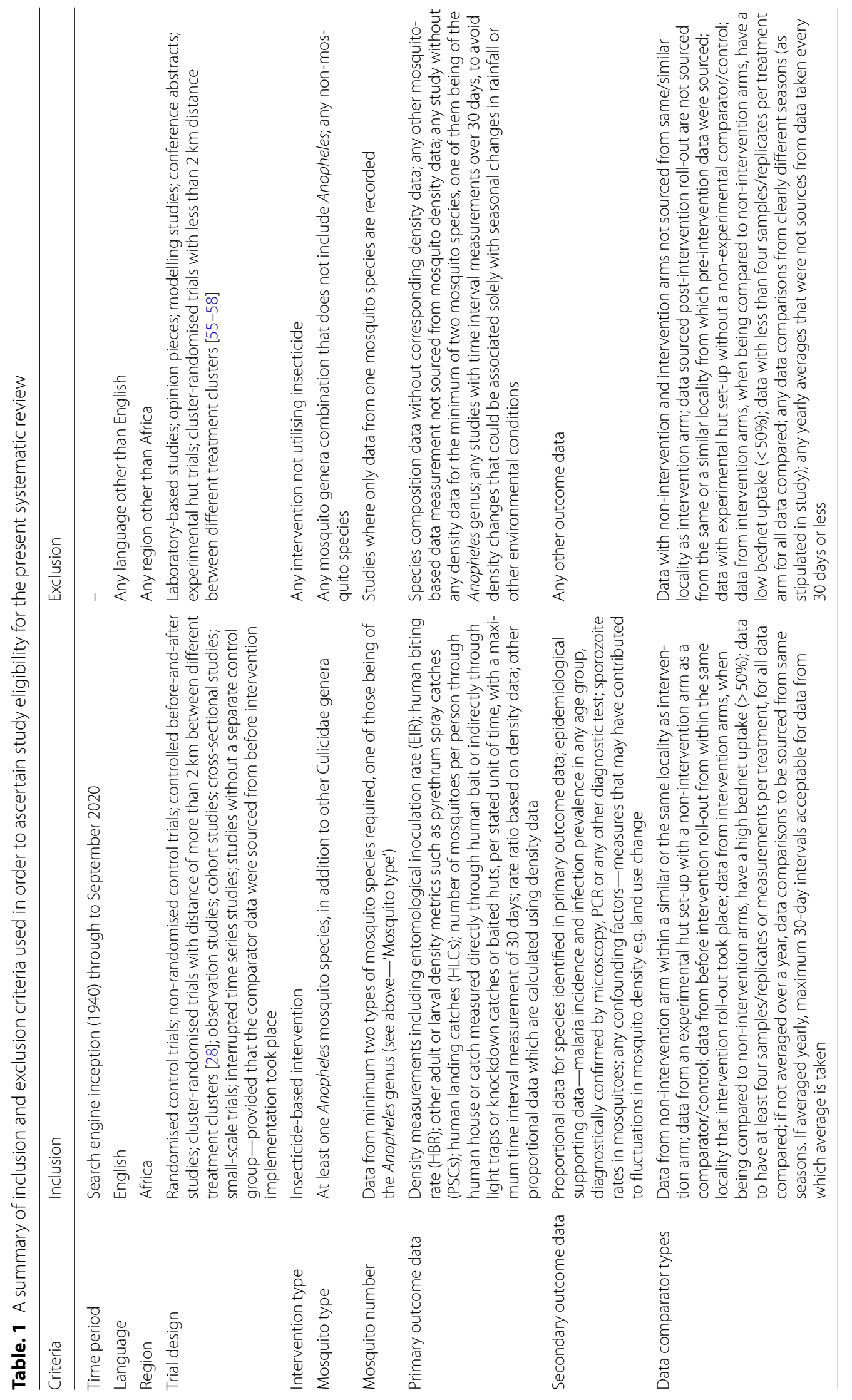


(indoors or outdoors), intervention and density. Some studies consisted of multiple density or malaria transmission observations for the stratifications. To differentiate between data arising from these multiple individual observations within studies and overall data arising from studies, data involving the number of such observations within studies is denoted by the terms 'observations within studies' or 'individual observations' Multiple observations within a study either fell into the same density category or were heterogeneous for density categories.

\section{Synthesis and statistical tests}

After scrutiny of the quality and quantity of extracted data, a mixed narrative and quantitative approach was adopted for results synthesis. Statistical test application to the dataset was limited due to the heterogeneity of data observations within and between individual studies, such as varieties of vector species identified and multiple sources of mosquito collection (indoors and outdoors, trap collection or human landing catches [HLCs]). Considering that some studies reported multiple data observations (e.g. data from two different sampling methods or intervention types), any statistical comparisons were made between individual data observations from each study as opposed to whole studies, in order to avoid exclusion of any data.

Only studies showing statistically significant density data were included in the review. Statistical tests forming the basis of the meta-analysis consisted of Chisquare tests of independence that considered categorical variables such as intervention type, insecticide use and insecticide resistance status, and rates of transmission. A Bonferroni adjustment was applied to the result, to minimise the potential for type I error [29]. Mean transmission intensity change differences between study characteristics such as insecticide resistance were assessed using a one-way analysis of variance (ANOVA).

When extracting and assessing vector density data, to avoid bias, all fluctuations in density that were recorded in each study were considered. For example, if a study took either a 'before-and-after intervention' data collection approach, or a longitudinal data collection approach, or a combination of both, all data satisfying inclusion criteria were extracted and assessed statistically and/or qualitatively.

Where only a proportion of longitudinal data or 'before-and-after intervention' data from within the same study involved category ID, the entire study was assigned to category ID. Transmission data that were tested statistically could be sourced from either sporozoite rates or clinical incidence and prevalence data, with pooled results termed 'transmission changes'. These data were assessed as individual observations rather than as whole studies, as some studies measured multiple sporozoite rates and therefore had multiple transmission observations.

All statistical testing was carried out using IBM SPSS version 27 software. Narrative analysis was carried out in accordance with the 'Synthesis without meta-analysis (SWiM) in systematic reviews: reporting guideline' [30].

\section{Results}

From 5569 publications screened for this review, 30 studies were eligible for quantitative and qualitative assessment (see Additional file 1: Tables S2 and S3). The review and selection process are detailed in Fig. 1.

\section{Quantitative analysis \\ Summary of density changes in response to interventions}

Of the 30 studies selected for analysis, in total there were 50 and 23 data observations on changes to density data or malaria transmission intensity data ('transmission data'), respectively. Of these, 66\% $(n=33), 2 \%(n=1)$ and $32 \%(n=16)$ of individual density data observations were assigned to category D I and ID, respectively. A significant relationship was found between insecticide type used and category, where use of a pyrethroid was more likely to result in a category ID density change (Table 2).

\section{Insecticide resistance data compared with transmission data}

A Chi-square test of independence revealed no significant relationship between insecticide resistance status and category of change in malaria transmission (increased, decreased or no change), $X^{2}=0.049, d f=1$, $P=0.825$. Similarly, there were no statistically significant differences in mean transmission between the two insecticide resistance categories (insecticide resistance reported, or not reported) (one-way ANOVA, $\left.F_{(1,21)}=17.401, P=0.945\right)$.

\section{Transmission data compared with density data}

Only a subset of studies that were selected for final analysis recorded transmission data, as this was not a primary observation required for review as per study eligibility criteria. Where category $\mathrm{D}$ individual observations within studies had monitored transmission $(n=12)$, average percentage transmission reduction was $48.6 \%$ (95\% CI 1-96.2). In category ID $(n=11)$, average percentage transmission reduction was $54.8 \%$ (95\% CI 30.2-79.4). There were no statistically significant differences between category mean transmission data as determined by oneway $\operatorname{ANOVA}\left(F_{(1,21)}=222.709, P=0.805\right)$. 


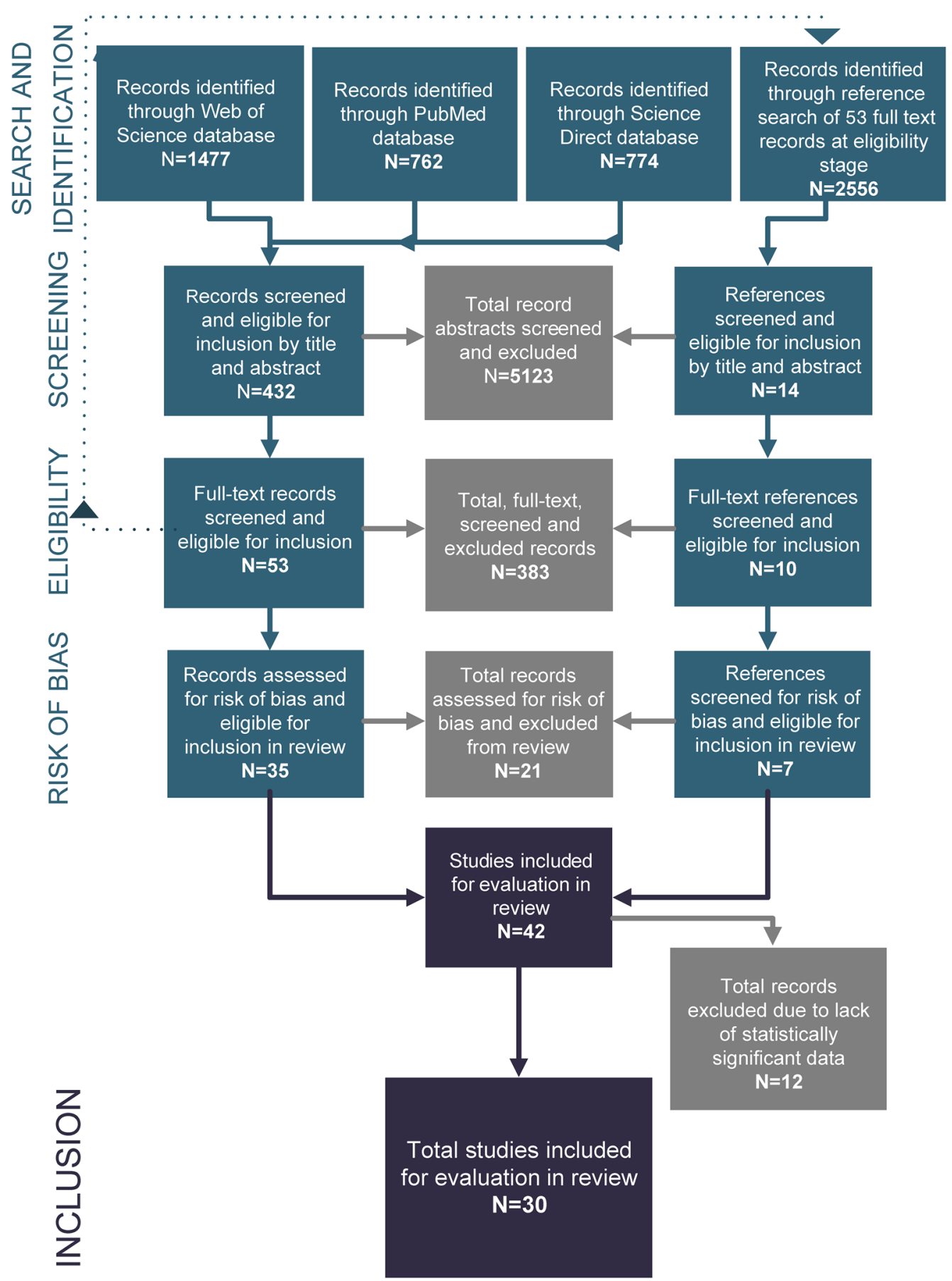

Fig. 1 Flowchart summary of numbers of publications identified from search engines and excluded or included at each subsequent stage of the analysis

\section{Qualitative assessment}

Qualitative assessment of category ID studies led to the dismissal of five studies from further consideration, as these were judged to provide only weak evidence for competitive release, such as marginal effects on population densities, or possible alternative explanations that could account for changes in vector species composition such as insecticide resistance or changes in rainfall (see Additional file 1). The remaining five category ID studies were judged to provide more compelling evidence 
Table 2 A summary of Chi-square comparisons of study characteristics and density change categories

\begin{tabular}{|c|c|c|c|c|}
\hline \multirow[t]{2}{*}{ Study characteristics } & \multicolumn{3}{|c|}{ Number of observations within category studies (\%) } & \multirow[t]{2}{*}{ Chi-square tests of independence } \\
\hline & Category D & Category 1 & Category ID & \\
\hline \multicolumn{5}{|l|}{ Intervention type } \\
\hline ITNs or LLINs & $10(30.4)$ & $1(100)$ & $12(75)$ & \multirow[t]{3}{*}{$X^{2}=11.564, d f=4, P=0.021^{b}$} \\
\hline IRS & $16(48.4)$ & - & $1(6.25)$ & \\
\hline Other ${ }^{\mathrm{a}}$ & $7(21.2)$ & - & $3(18.75)$ & \\
\hline \multicolumn{5}{|l|}{ Insecticide type } \\
\hline Pyrethroids & $16(48.5)$ & $1(100)$ & $15(93.7)$ & \multirow[t]{2}{*}{$X^{2}=10.157, d f=2, P=0.006^{b *}$} \\
\hline Non-pyrethroids & $17(51.5)$ & - & $1(6.3)$ & \\
\hline \multicolumn{5}{|l|}{ Insecticide resistance } \\
\hline Resistance & $18(55)$ & $1(100)$ & $7(43.8)$ & \multirow[t]{2}{*}{$X^{2}=1.445, d f=2, P=0.486^{b}$} \\
\hline No resistance & $15(45)$ & - & $9(56.2)$ & \\
\hline \multicolumn{5}{|l|}{ Malaria transmission } \\
\hline Increased transmission & $2(16)$ & - & $1(9)$ & \multirow[t]{2}{*}{$X^{2}=0.290, d f=1, P=0.590^{c}$} \\
\hline Decreased transmission & $10(84)$ & - & $10(91)$ & \\
\hline \multicolumn{5}{|l|}{ Collection methods } \\
\hline Indoor & $24(72)$ & - & $10(55.7)$ & \multirow[t]{3}{*}{$X^{2}=5.705, d f=4, P=0.222^{b}$} \\
\hline Outdoor & $3(10)$ & - & $3(16.6)$ & \\
\hline Indoor + Outdoor & $6(18)$ & $1(100)$ & $5(27.7)$ & \\
\hline
\end{tabular}

a Push-pull systems, hessian strips, long-lasting insecticide-treated blanket, insecticide-treated bednet plus sisal curtains, ITN plus LLIN combinations

${ }^{\mathrm{b}}$ Bonferroni-adjusted $P<0.0125, n=50$

'Bonferroni-adjusted $P<0.016, n=23$

${ }^{*}$ Represents a significant difference

that release from competition could have contributed to changes in species composition due to release from competition and are described below.

Sougoufara et al. [31] examined the impact of the introduction in 2008 of LLINs on densities of the three sympatric species of the An. gambiae complex in Dielmo, Senegal. In 2006, prior to LLIN introduction, An. coluzzii, An. gambiae, and An. arabiensis represented 57\%, 19\% and 21\% of Anopheles captured in human landing catches (HLCs), and accounted for 4.33, 1.45 and 1.62 bites per person per night (bpn), respectively. By 2008, with the introduction of LLINs in July, the three species represented $14 \%, 3 \%$ and $83 \%$ of proportions of HLCs and had densities of 2.00, 0.44 and $12.12 \mathrm{bpn}$. Changes in rainfall alone could not account for this change in species densities, as precipitation in 2008 was $834.4 \mathrm{~mm}$ and in 2006, $583.4 \mathrm{~mm}$ [32]. Indeed, the heavier rainfall observed in 2008 should have favoured higher densities of $A n$. coluzzii and An. gambiae s.s., as both species are typically most abundant in the wet season in Dielmo [31].

Over this same time frame, the prevalence of malaria in children under 14 years of age declined from 36.8 to $12.3 \%$, and from 27.6 to $9.0 \%$ for those aged over 15 years, presumably reflecting the shift in composition of the complex to the less endophilic, anthropophilic $A n$. arabiensis. By 2010, there was a rebound in the numbers of An. gambiae, but not of An. coluzzii, which continued to show a decline in density. Although the absolute density of An. arabiensis was lower in 2010 than in 2008, it remained the most abundant species, with An. coluzzii, An. gambiae and An. arabiensis accounting for 8, 31 and $60 \%$, respectively, of HLC samples. This rebound of An. gambiae s.s. may have been as a result of the emergence of insecticide resistance, as increases in pyrethroid resistance had been reported in the area in An. gambiae sensu lato (s.l.), although individual species of the complex were not specifically investigated. Adherence to bednet usage may have also contributed to this rebound, as selfreported LLIN usage in Dielmo reduced from $75.6 \%$ in 2008 [32] to $58.3 \%$ in 2010 [31].

Russell et al. [33] also reported a shift in the composition of the An. gambiae complex from An. gambiae s.s. to An. arabiensis. In that study, the impact of the 2006 introduction of insecticide-treated nets (ITNs) in rural Tanzania [24] was examined for the period between 2004 and 2009 [33]. Using the absolute density data of the $A n$. gambiae s.l. reported in Russell et al. [33] for 2004 and 2009, and relative density data for An. gambiae s.s. and An. arabiensis reported in the intervention area for 2004 [34, 35] and 2009 [33], the changes in absolute densities of An. gambiae s.s. and An. arabiensis over this period were calculated here. These data confirmed an almost 
complete reversal in the absolute densities of An. gambiae s.s. and An. arabiensis between 2004 and 2009 for this Tanzanian study (Table 3). Moreover, the authors showed that this shift in species composition was not related to rainfall patterns in the area.

Zhou et al. [36] reported similar, although less dramatic, observations concerning an increase in density of An. arabiensis following decreased density of An. gambiae s.s. They examined the entomological impacts of the introduction of ITNs in 2006 in villages in Western Kenya between 2002 and 2010. In Kombewa, where the main vectors were $A n$. gambiae s.l. and An. funestus s.l., mosquito density decreased by $90 \%$ from 2002 to 2007 . The 2003 density of An. gambiae s.l. was 1.3 females per house per night (fhn), with $1.7 \%$ of those samples being An. arabiensis. In 2009, An. gambiae s.l. accounted for $0.23 \mathrm{fhn}, 61.7 \%$ being An. arabiensis.

These data allowed the calculation here of the absolute density of An. gambiae s.s., which decreased more than 14-fold from $1.278 \mathrm{fhn}$ in 2003 to $0.088 \mathrm{fhn}$ in 2009. Over the same time frame, there was more than a sixfold increase in the absolute density of An. arabiensis, from 0.022 to $0.142 \mathrm{fhn}$. In Iguhu, An. gambiae s.s. decreased more than 11-fold from $2.178 \mathrm{fhn}$ in 2003 to $0.195 \mathrm{fhn}$ in 2006, whereas An. arabiensis doubled in density from 0.022 to $0.045 \mathrm{fhn}$ over the same period.

Bayoh et al. [37] also examined the species composition of An. gambiae s.l. in two regions in western Kenya: Asembo, where ITN ownership was circa 90\% from 1999 onwards, and neighbouring Seme, where ITN coverage was below $5 \%$ in 1999, but gradually increased to circa $30 \%$ in 2003 and was greater than $70 \%$ by 2007 . In these communities, An. gambiae s.s. and An. arabiensis were the two dominant malaria vectors, with $A n$. gambiae s.s. predominating until 1999. After 1999, the proportion of An. gambiae s.s. relative to An. arabiensis steadily declined, so that by 2009 the latter accounted for circa 99\% of indoor pyrethrum spray catches (PSCs) and the former $1 \%$. These changes could not be accounted for by rainfall.

Consistent with this proportional data from adult collections, in 2003, the absolute larval density of $A n$. gambiae s.s. in Seme, with low ITN coverage, was higher than that of An. arabiensis and of that found in Asembo, where ITN coverage was high. As ITN coverage increased in Seme between 2003 and 2009, the relative proportion of An. gambiae s.s. to An. arabiensis obtained in larval collections declined. Thus, the evidence regarding competitive releases of species from this study was consistent with that of Sougoufara et al. [31], Russell et al. [33] and Zhou et al. [36].

Gillies and Smith [38] reported on the impacts on the An. funestus group from the application of IRS using dieldrin in the Kihurio village in the South Pare region of Tanganyika (now Tanzania) in East Africa. Before spraying in November 1955, endophilic, anthropophilic An. funestus predominated seasonally in box shelter catches, while numbers of exophilic, zoophilic An. rivulorum were considerably lower (Fig. 2).

After spraying, from January 1956, no sample from box catches was identified as An. funestus, while there were substantial increases in the seasonal density of the population of An. rivulorum. Although increases in vector densities showed marked seasonal variation (Fig. 2), the shift in species composition persisted for more than 2 years, indicating that such changes in species composition were unlikely to be from environmental or rainfall conditions associated with a particular season. Nor were there any reported changes in irrigation in Kihurio over this time. Anopheles rivulorum was identified by the presence of 'two spots' on its fifth wing vein, which were never found in An. funestus [38]. The proportion of An. rivulorum displaying this phenotype was reported to vary from region to region, so it was possible that the proportion displaying 'two spots' changed over the course of the study. However, while this could have exaggerated the magnitude of the effect from the shift in population densities, it would not alone account for the substantial increase in density of An. rivulorum.

The authors also reported overlap, albeit incomplete, in the aquatic habitats of An. rivulorum and An. funestus, found in flooded rice paddies and irrigation channels. Anopheles funestus, but not An. rivulorum, was also reported to occupy stagnant aquatic

Table. 3 Evidence from Russell et al. [33] for increases in the absolute population densities of An. arabiensis concomitant with decreases in absolute densities of An. gambiae following roll-out of LLINs in 2006 [24]

\begin{tabular}{|c|c|c|c|}
\hline Measurement & Species & 2004 & 2009 \\
\hline Absolute density \pm standard error (bpn) & An. gambiae s.l. & $0.660 \pm 0.006$ & $0.575 \pm 0.008$ \\
\hline \multirow[t]{2}{*}{ Relative proportions \pm standard error (\%) } & An. gambiae s.s. & $94.7 \pm 0.05^{\mathrm{a}}$ & 0.5 \\
\hline & An. arabiensis & $5.3 \pm 0.05^{\mathrm{a}}$ & 99.5 \\
\hline \multirow[t]{2}{*}{ Absolute density, calculated using relative proportion (bpn) } & An. gambiae s.s. & 0.625 & 0.003 \\
\hline & An. arabiensis & 0.035 & 0.572 \\
\hline
\end{tabular}

${ }^{a}$ Data from Scholte [34] and Scholte et al. [35] 


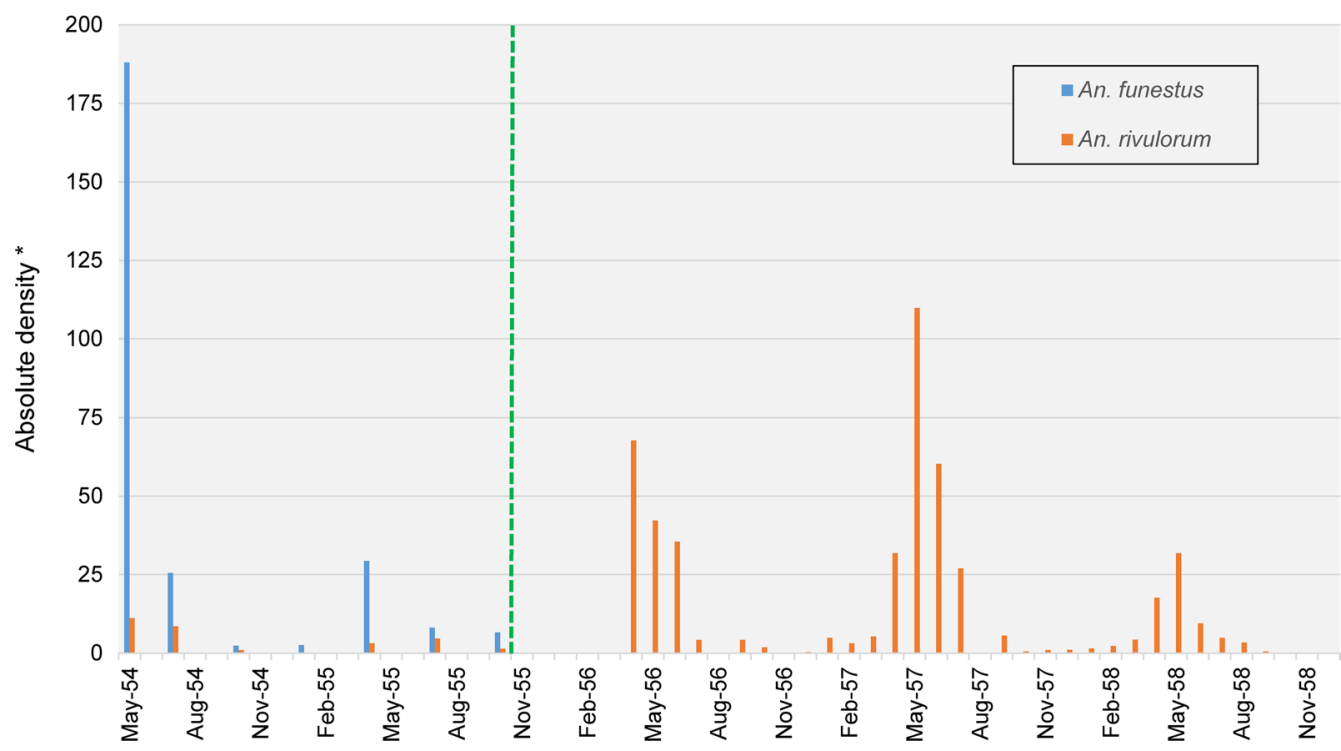

Fig. 2 Replacement of An. funestus by An. rivulorum in Kihurio, South Pare Area, Tanganyika Territory (modern day Tanzania), East Africa, following IRS with dieldrin in November 1955. Data were extracted from Tables I and II to reproduce with permission Fig. 1 in Gillies and Smith [38]. Green dashed line shows when IRS occurred. No samples of An. funestus were recorded after January 1956. *Proxies for absolute density were recorded as average monthly catch of mosquitoes from the resting boxes that were used as traps throughout the study area, the number of which varied from day to day. The number of catches per month also varied. Density was therefore calculated using the unit'20 box/days', which represented the average catch of mosquitoes from outdoor traps in the study area on any one day in a month, which allowed comparison between data at different time points

habitats. After spraying, the authors did not observe the colonisation of any stagnant water sites by $A n$. rivulorum. Rather, they speculated that interspecific competition in overlapping aquatic habitat sites may have accounted for the relative rarity of $A n$. rivulorum before spraying. With the near elimination of $A n$. funestus following IRS, An. rivulorum could have been released from competition, causing such a dramatic expansion in its numbers.

Gillies and Smith [38] also noted that there was a second possibility for release from competition. Other studies in the area had previously reported on the reduction in population density of An. gambiae, in addition to An. funestus [39]. Anopheles gambiae was also found to overlap with An. rivulorum in aquatic habitats, so some competitive interactions could be envisaged. However, the annual peak numbers of $A n$. gambiae were from January to March, whereas both An. funestus and An. rivulorum peaked seasonally between April and June. This supports the argument that the increases in density of An. rivulorum were caused by the elimination of An. funestus leading to the release of $A n$. rivulorum from competition in aquatic habitats. Despite its increased population density, $A n$. rivulorum did not contribute to malaria transmission in the area, because it was principally zoophilic.

\section{Discussion}

The aim of this systematic review was to search for evidence indicating the potential for vector species to be released from competition following insecticide-based vector control of Anopheles species in malaria-endemic sub-Saharan Africa. Of necessity, studies that were included in this review reported population density data for at least two species of mosquito, with at least one belonging to the Anopheles genus, both before and after, or with and without, intervention implementation (Table 1).

The stringency of inclusion and exclusion criteria meant that some studies that have previously been cited $[1,20]$ as providing evidence for release of vector species from competition following insecticide use in Africa, such as Gillies and Furlong [40], were identified but excluded from this analysis because they provided insufficient data on densities of both insecticide-targeted species and other vector species.

Although all category ID studies provided some degree of evidence that opposing changes in species densities were consistent with the release of other vector species from competition (Fig. 3a), it is also important to consider alternative explanations. Competitive interactions between targeted Anopheles species and other vector species could also occur through indirect effects where 


\begin{tabular}{|l|l|l|l|}
\hline \multicolumn{1}{c}{ Scenario } \\
$\begin{array}{l}\text { a Differential effects } \\
\text { on species from } \\
\text { insecticide with } \\
\text { release from } \\
\text { competition. }\end{array}$
\end{tabular}

Fig. 3 Possible scenarios for changes in densities of Anopheles species observed in studies from category ID. Intervention involves an insecticide-based vector control measure targeting vector species $V$ resulting in its decreased population density, concomitant with increased population density of other vector species $D$. White circles represent population densities. a Differential effects on species from insecticide with release from competition. b Release from apparent asymmetric competition via impacts on predator-prey interactions. Black lines represent competitive pressure on a species at the tip of arrowheads, with ' + 'indicating a positive effect and ' ++ ' indicating an even stronger positive effect, while'-'indicates a negative effect and '- -'a stronger negative effect. ' 0 ' indicates negligible competitive effects. Effects shown in parentheses indicate apparent competitive effects [41]. c Species-specific insecticide resistance with release from competition. $\mathbf{d}$ Species-specific insecticide resistance with increase in habitat resources. The ${ }^{\mathbb{R}}$ superscript after $D$ indicates insecticide resistance in that species. e Differential impacts on species from insecticide with increase in habitat resources. Blue arrows indicate potentially positive effects on population densities from increases in habitat resources, such as increased rainfall. $\mathbf{a}$ and $\mathbf{b}$ adapted from [54]

two species could be in apparent asymmetric competition with each other via predator-prey relationships [41], with a differential impact of the insecticide on one species leading to an increase in density of the other species (Fig. 3b). In theory, in such a scenario the two species would not need to share habitats, but would need 
to share predators that could, for example, visit separate larval habitats. Both species would of course still need to co-locate in the same vicinity to be preyed upon by a shared predator.

Insecticide resistance could also influence changes in species composition. Indeed, Helinski et al. [42] and Zhou et al. [43] reported that reduced susceptibility to the insecticide was the most likely cause of divergent changes in species densities resulting from insecticide use. As a result, these category ID studies were excluded from the present qualitative assessment. However, even if a species was fully resistant to an insecticide, its density should only be maintained, and not increase, in the presence of the insecticide, unless additional factors are also influencing species composition, such as release from competition (Fig. 3c) or increases in habitat resources (Fig. 3d). In support of this, we found that instances of insecticide resistance were reported for similar proportions of observations across all density categories (55 and $44 \%$ in categories D and ID, respectively), and there was no statistically significant relationship between insecticide resistance status and category of density change.

Another possible explanation to account for changes in species composition could involve differential effects of the insecticide on one species over another, combined with an increase in habitat resources, such as increased rainfall or changes in irrigation practices that might augment aquatic habitats. The less affected species could then increase in number while the other species reduced in density, without the need to invoke any explanation involving interspecific competition (Fig. 3e). Some studies did attempt to address this scenario by searching for evidence for altered patterns of rainfall or irrigation that could support species composition changes, finding none $[31,38]$, while one was excluded from the narrative analysis because the reported evidence was judged to be consistent with changes in species composition being driven by changes in rainfall [44].

The most common interventions in category ID studies involved LLINs or ITNs. Species that are endophilic and anthropophagic are behaviourally vulnerable to indoor insecticide-based vector control measures such as IRS and LLINs, which can thus lead to their effective suppression. In the 10 category ID studies identified, there was heterogeneity in species that increased or decreased in density. Anopheles arabiensis, a species known to exhibit behavioural plasticity in response to vector control, was found to increase in density in four of these studies but decrease in none. By contrast, An. funestus, which shows less behavioural plasticity and has more fixed habitat requirements [45], decreased in density in four of these studies and increased in one. A greater ability to display behavioural evasion of endophilic and anthropophagic needs could thus lead to residual disease transmission. Indeed, mosquito taxa exhibiting such evasive traits may be more accurately described as behaviourally resilient rather than insecticide-resistant [8]. Thus, for each species there is likely to be an interplay between physiological status towards insecticides and behavioural plasticity that together determine the impact of the intervention.

Limitations also surround the diversity in methods of mosquito collection between each study. Whilst many studies collected both indoor and outdoor samples of mosquitoes, some only performed indoor collections. This may be problematic to interpret, due to the behaviourally selective nature of indoor collections, likely to influence the balance of species densities indoors. For example, An. gambiae s.s. tends to bite at night indoors and then rest on the walls of the dwelling and so is likely to be overrepresented in indoor resting catches compared with less endophilic species. However, results indicated that density change type was not influenced by mosquito collection location. Nonetheless, where indoor and outdoor data were reported separately rather than averaged, one study showed that density changes seen from indoor collections (category ID) differed from those seen in outdoor collections (category D) [46], and two studies showed data consistent with density change type between indoor and outdoor collections $[47,48]$.

Two additional factors may have contributed to the substantial variation in the strength of evidence supporting release of other vector species from competition found in category ID studies. Firstly, it is known that there can be heterogeneity in observed larval densities in aquatic habitats in the field [13]. This could lead to variability in the propensity for competition between vector species to occur, which could be observed as variability in the frequency and effect size of any releases of other vector species from competition following population suppression of Anopheles species. Secondly, release of other vector species from competition could vary temporally. Some releases might only be temporary and occur over relatively short time frames. For example, should LLIN use decline in human behavioural responses to reductions in populations of targeted species, the density of that targeted species could again increase to reduce or reverse any effects of release from competition on the densities of other vector species [31, 32]. Thus, densities reported in any one study may not have reflected maximal levels of differences in density changes between Anopheles species and other disease vectors.

Despite the considerable heterogeneity between category ID studies, potentially involving complex interactions between habitat, climate, environment and land use changes that may have further contributed to changes in species composition, qualitative analysis 
identified five studies reporting significant opposing increases and decreases in vector species that were considered to provide compelling evidence that it was possible for competitive release to occur following insecticide-based population suppression of Anopheles vector species [31, 33, 36-38]. In all five cases, highly effective malaria vectors appeared to be replaced with less efficient vectors of malaria transmission. Moreover, in the two of these studies that had also reported on data relevant to disease incidence, indicators of malaria transmission also decreased [31, 36], suggesting that release of vector species from competition did not increase disease transmission under those conditions.

Aquatic habitats for mosquito larval development are believed to have the most impact on adult population numbers $[2,3]$. Prerequisites, therefore, for direct interspecies competitive interactions between mosquito species are that they are both sympatric and share the same aquatic habitats. Indeed, it is noteworthy that for the five most compelling studies identified in this review, putative competitor species of insecticide-targeted Anopheles species were invariably sibling species. Sibling species An. gambiae s.s. and An. arabiensis are known to share larval habitats, and competitive interactions between these species have been observed [11], supporting an association between changes in species composition and competitive release [33, 36, 37]. Anopheles coluzzii and An. arabiensis have also been reported to share aquatic habitats, adding weight to the conclusions of Sougoufara et al. [31] that reductions in the density of An. coluzzii could have released An. arabiensis from competition. Similarly, Gillies and Smith [38] found that An. rivulorum replaced it sibling species An. funestus following IRS, and it was reported that the two species shared larval habitats.

Consistent with such observations, this review found no convincing evidence that insecticide-based population suppression of An. gambiae s.l. could lead to increases in the densities of species outside of the Anopheles genus, such as Aedes aegypti, a major vector species responsible for the transmission of dengue and yellow fever. Indeed, Ae aegypti and An. gambiae s.l. do not share aquatic habitats, with the former favouring water tanks, discarded containers, tyres, shells or tree holes [49] and the latter preferring natural aquatic habitats ranging from small, clean, sunlit, ephemeral ones that lack vegetation to larger, riparian, more permanent bodies of water, typically with overhanging vegetation, such as riverbanks with slow-moving water or irrigation channels of rice paddies [50]. Moreover, Ae. aegypti is considered an urban dweller whereas $A n$. gambiae occupies rural locations.

\section{Conclusions}

While it cannot be asserted that the evidence obtained by this review is representative of entomological sequelae from the use of insecticide-based vector control in the field, at least five studies provided persuasive evidence that insecticide use could, at least under some circumstances, lead to competitive release of non-targeted vector species. These observations should therefore form part of the considerations relating to integrated vector management approaches for malaria control in Africa [51]. More broadly, novel forms of vector control that could in the future effectively target malaria vector species $[52,53]$ might also result in increases in the densities of non-targeted, sympatric vector species that share larval habitats or predators, at least under certain circumstances. Hence, such interventions could effectively reduce the incidence of malaria and thus overall disease burden even against the background of competitive releases of other non-targeted, sympatric vector species.

\section{Abbreviations}

bpn: Bites per person per night; fhn: Females per house per night; Category D: Density of all species decreased with use of insecticide-based vector control; Category I: Density of all species increased with use of insecticide-based vector control; Category ID: Density of one or more vector species increased while the density of another one or more species decreased with use of insecticidebased vector control; HLC: Human landing catch; IRS: Indoor residual spraying; ITN: Insecticide-treated bednet; LLIN: Long-lasting insecticide-treated bednet; LTC: Light trap collection; PSC: Pyrethrum spray catch; UTN: Untreated bednet.

\section{Supplementary Information}

The online version contains supplementary material available at https://doi. org/10.1186/s13071-021-04975-0.

Additional file 1. Risk of bias analysis. Table S1. A summary of the results of risk of bias assessments undertaken on each individual study. Table S2. Studies with statistically significant changes in absolute densities of vector species. Table S3. Studies without statistically significant changes in absolute densities of vector species. Category ID studies dismissed from qualitative assessment in this review, as providing only weak evidence for competitive release or suggesting alternative explanations for changes in vector species composition.

Additional file 2: Dataset S1. Vector population density and malaria transmission data from the 30 studies selected for inclusion in this study.

Acknowledgements

We thank Camilla Beech, Austin Burt, Mamadou Coulibaly, Silke Fuchs, Charles Godfray, Talya Hackett, Andrew McKemey, John Mumford, Samantha O'Loughlin, Seynabou Sougoufara, Frederic Tripet, Geoff Turner, and Katie Willis for useful input over the course of this study.

\section{Authors' contributions}

$\mathrm{AQ}$ and JBC developed the study design and wrote the manuscript. AQ performed the literature searches and conducted screening of abstracts. Both $A Q$ and JBC screened full-text articles and assessed selected articles for risk of bias. AQ and JBC read, summarised, and categorised all literature selected from searches. $A Q$ and $J B C$ performed statistical and quantitative analyses of the data. AQ and JBC generated the figures and tables. Both authors read and approved the final manuscript. 


\section{Funding}

Both authors are members of the Target Malaria not-for-profit research consortium, which receives core funding from the Bill \& Melinda Gates Foundation and Open Philanthropy.

\section{Availability of data and materials}

All data and material are presented in the manuscript and supplementary material.

\section{Declarations}

Ethics approval and consent to participate

Not applicable.

\section{Consent for publication}

Not applicable.

\section{Competing interests}

Both authors are part of the Target Malaria not-for-profit research consortium, which aims to develop novel malaria vector control tools that complement existing insecticide-based vector control interventions. JBC is an employee of Imperial College London, whose role for the Target Malaria not-for-profit research consortium is funded by the Bill \& Melinda Gates Foundation, and who has received travel grants from the Foundation for the National Institutes of Health. AQ is an employee of Imperial College London, whose role is funded by the Open Philanthropy.

\section{Received: 6 March 2021 Accepted: 23 August 2021}

Published online: 08 September 2021

\section{References}

1. Lounibos LP. Competitive displacement and reduction. J Am Mosq Control Assoc. 2007;23(Suppl. 2):276-82.

2. Muriu SM, Coulson T, Mbogo CM, Godfray HC. Larval density dependence in Anopheles gambiae s.S., the major African vector of malaria. J Anim Ecol. 2013;82:166-74.

3. Barreaux AMG, Stone CM, Barreaux P, Koella JC. The relationship between size and longevity of the malaria vector Anopheles gambiae (s.s.) depends on the larval environment. Parasit Vectors. 2018;11:485.

4. Sinka ME, Bangs MJ, Manguin S, Coetzee M, Mbogo CM, Hemingway J, et al. The dominant Anopheles vectors of human malaria in Africa, Europe and the Middle East: occurrence data, distribution maps and bionomic precis. Parasit Vectors. 2010;3:117.

5. Sougoufara S, Doucoure S, Backe Sembene PM, Harry M, Sokhna C. Challenges for malaria vector control in sub-Saharan Africa: resistance and behavioral adaptations in Anopheles populations. J Vector Borne Dis. 2017:54:4-15.

6. Doucoure S, Thiaw O, Wotodjo AN, Bouganali C, Diagne N, Parola P, et al. Anopheles arabiensis and Anopheles funestus biting patterns in Dielmo, an area of low level exposure to malaria vectors. Malar J. 2020;19(1):230.

7. Sougoufara S, Diedhiou SM, Doucoure S, Diagne N, Sembene PM, Harry $\mathrm{M}$, et al. Biting by Anopheles funestus in broad daylight after use of longlasting insecticidal nets: a new challenge to malaria elimination. Malar J. 2014;13:125.

8. Govella NJ, Chaki PP, Killeen GF. Entomological surveillance of behavioural resilience and resistance in residual malaria vector populations. Malar J. 2013:12:124.

9. Coetzee M, Craig M, le Sueur D. Distribution of African malaria mosquitoes belonging to the Anopheles gambiae complex. Parasitol Today. 2000;16:74-7.

10. Chen H, Minakawa N, Cui L, Yan G. Conspecific sharing of breeding sites by Anopheline female mosquitoes (Diptera: Culicidae) inferred from microsatellite markers. J Insect Behav. 2007;21:24-33.

11. Paaijmans KP, Huijben S, Githeko AK, Takken W. Competitive interactions between larvae of the malaria mosquitoes Anopheles arabiensis and Anopheles gambiae under semi-field conditions in western Kenya. Acta Trop. 2009;109:124-30.
12. Kirby MJ, Lindsay SW. Effect of temperature and inter-specific competition on the development and survival of Anopheles gambiae sensu stricto and An. arabiensis larvae. Acta Trop. 2009;109(2):118-23.

13. Majambere S, Lindsay SW, Green C, Kandeh B, Fillinger U. Microbial larvicides for malaria control in The Gambia. Malar J. 2007:6:76.

14. James SL, Marshall JM, Christophides GK, Okumu FO, Nolan T. Toward the definition of efficacy and safety criteria for advancing gene drive-modified mosquitoes to field testing. Vector Borne Zoonotic Dis. 2020:20:237-51.

15. Wangdi K, Furuya-Kanamori L, Clark J, Barendregt JJ, Gatton ML, Banwell

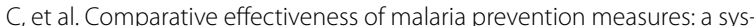
tematic review and network meta-analysis. Parasit Vectors. 2018;11:210.

16. WHO. World malaria report 2020: 20 years of global progress and challenges.

17. Juliano SA, Philip LL. Ecology of invasive mosquitoes: effects on resident species and on human health. Ecol Lett. 2005:8:558-74.

18. Trapido H, Aitken TH. Study of a residual population of Anopheles $L$. labranchiae Falleroni in the Geremeas Valley, Sardinia. Am J Trop Med Hyg. 1953;2:658-76.

19. Gilotra SK, Rozeboom LE, Bhattacharya NC. Observations on possible competitive displacement between populations of Aedes aegypti Linnaeus and Aedes albopictus Skuse in Calcutta. B World Health Organ. 1967;37:437-46.

20. Collins CM, Bonds JAS, Quinlan MM, Mumford JD. Effects of the removal or reduction in density of the malaria mosquito, Anopheles gambiae s.l. on interacting predators and competitors in local ecosystems. Med Vet Entomol. 2019;33:1-15.

21. Mbogo CN, Baya NM, Ofulla AV, Githure Jl, Snow RW. The impact of permethrin-impregnated bednets on malaria vectors of the Kenyan coast. Med Vet Entomol. 1996;10:251-9.

22. Mwangangi JM, Mbogo CM, Orindi BO, Muturi EJ, Midega JT, Nzovu $J$, et al. Shifts in malaria vector species composition and transmission dynamics along the Kenyan coast over the past 20 years. Malar J. 2013;12:13.

23. Mutuku FM, King CH, Mungai $P$, Mbogo C, Mwangangi J, Muchiri EM, et al. Impact of insecticide-treated bed nets on malaria transmission indices on the south coast of Kenya. Malar J. 2011;10:356.

24. Russell TL, Lwetoijera DW, Maliti D, Chipwaza B, Kihonda J, Charlwood JD, et al. Impact of promoting longer-lasting insecticide treatment of bed nets upon malaria transmission in a rural Tanzanian setting with preexisting high coverage of untreated nets. Malar J. 2010;9:187.

25. Moher D, Liberati A, Tetzlaff J, Altman DG, Group P. Preferred reporting items for systematic reviews and meta-analyses: the PRISMA statement. BMJ. 2009:339:b2535.

26. Connolly JB, Qureshi A. Systematic review of evidence on the potential for insecticide-based control of the human malaria vector An. gambiae to release other disease vectors from competition. PROSPERO 2020;CRD42020194304.

27. PROSPERO, International prospective register of systematic reviews. https://www.crd.york.ac.uk/PROSPERO/. Accessed 22 Aug 2021

28. Verdonschot PFM, Besse-Lototskaya AA. Flight distance of mosquitoes (Culicidae): a metadata analysis to support the management of barrier zones around rewetted and newly constructed wetlands. Limnologica. 2014;45:69-79

29. McGuinness LA, Higgins JPT. Risk-of-bias VISualization (robvis): an R package and Shiny web app for visualizing risk-of-bias assessments. Res Synth Methods. 2020;12:55-61.

30. Wright SP. Adjusted P-values for simultaneous inference. Biometrics. 1992;48:1005-13.

31. Sougoufara S, Harry M, Doucoure S, Sembene PM, Sokhna C. Shift in species composition in the Anopheles gambiae complex after implementation of long-lasting insecticidal nets in Dielmo. Senegal Med Vet Entomol. 2016;30:365-8.

32. Trape J-F, Tall A, Sokhna C, Ly AB, Diagne N, Ndiath O, et al. The rise and fall of malaria in a west African rural community, Dielmo, Senegal, from 1990 to 2012: a 22 year longitudinal study. Lancet Infect Dis. 2014:14:476-88.

33. Russell TL, Govella NJ, Azizi S, Drakeley CJ, Kachur SP, Killeen GF. Increased proportions of outdoor feeding among residual malaria vector 
populations following increased use of insecticide-treated nets in rural Tanzania. Malar J. 2011;10:80.

34. Scholte E. The entomopathogenic fungus Metarhizium anisopliae for mosquito control Impact on the adult stage of the African malaria vector Anopheles gambiae and filariasis vector Culex quinquefasciatus. Wageningen: Doctor of Philosophy, Wageningen University; 2004.

35. Scholte EJ, Ng'habi K, Kihonda J, Takken W, Paaijmans K, Abdulla S, et al. An entomopathogenic fungus for control of adult African malaria mosquitoes. Science. 2005;308:1641-2.

36. Zhou G, Afrane YA, Vardo-Zalik AM, Atieli H, Zhong D, Wamae P, et al. Changing patterns of malaria epidemiology between 2002 and 2010 in Western Kenya: the fall and rise of malaria. PLoS ONE. 2011;6:e20318.

37. Bayoh MN, Mathias DK, Odiere MR, Mutuku FM, Kamau L, Gimnig JE, et al. Anopheles gambiae: historical population decline associated with regional distribution of insecticide-treated bed nets in western Nyanza Province, Kenya. Malar J. 2010;9:62.

38. Gillies MT, Smith A. The effect of a residual house-spraying campaign in East Africa on species balance in the Anopheles funestus group. The replacement of A. funestus Giles by A. rivulorum Leeson. Bull Entomol Res. 1960:51:243-52

39. Smith A. Effect of residual house spraying in the plains of anopheline densities in huts on the Pare Mountains. Nature. 1959;183:198-9.

40. Gillies MT, Furlong M. An investigation into the behaviour of Anopheles parensis Gillies at Malindi on the Kenya coast. Bull Entomol Res. 1964:55:1-16.

41. Holt RD, Bonsall MB. Apparent competition. Annu Rev Ecol Evol Syst. 2017:48:447-71.

42. Helinski ME, Nuwa A, Protopopoff N, Feldman M, Ojuka P, Oguttu DW, et al. Entomological surveillance following a long-lasting insecticidal net universal coverage campaign in Midwestern Uganda. Parasit Vectors. 2015:8:458.

43. Zhou G, Lee MC, Githeko AK, Atieli HE, Yan G. Insecticide-treated net campaign and malaria transmission in Western Kenya: 2003-2015. Front Public Health. 2016:4:153.

44. Sougoufara S, Thiaw O, Cailleau A, Diagne N, Harry M, Bouganali C, et al. The impact of periodic distribution campaigns of long-lasting insecticidal-treated bed nets on malaria vector dynamics and human exposure in Dielmo, Senegal. Am J Trop Med Hyg. 2018;98:1343-52.

45. Nambunga IH, Ngowo HS, Mapua SA, Hape EE, Msugupakulya BJ, Msaky DS, et al. Aquatic habitats of the malaria vector Anopheles funestus in rural south-eastern Tanzania. Malar J. 2020;19:219.

46. Mmbando AS, Ngowo HS, Kilalangongono M, Abbas S, Matowo NS, Moore SJ, et al. Small-scale field evaluation of push-pull system against early- and outdoor-biting malaria mosquitoes in an area of high pyrethroid resistance in Tanzania. Wellcome Open Res. 2017;2:112.
47. Meyers JI, Pathikonda S, Popkin-Hall ZR, Medeiros MC, Fuseini G, Matias A, et al. Increasing outdoor host-seeking in Anopheles gambiae over 6 years of vector control on Bioko Island. Malar J. 2016:15:239.

48. Pinder M, Jawara M, Jarju LBS, Salami K, Jeffries D, Adiamoh M, et al. Efficacy of indoor residual spraying with dichlorodiphenyltrichloroethane against malaria in Gambian communities with high usage of long-lasting insecticidal mosquito nets: a cluster-randomised controlled trial. Lancet. 2015;385:1436-46.

49. Saleh F, Kitau J, Konradsen F, Alifrangis M, Lin CH, Juma S, et al. Habitat characteristics for immature stages of Aedes aegypti In Zanzibar City, Tanzania. J Am Mosq Control Assoc. 2018;34:190-200.

50. Diabate A, Dabire RK, Kim EH, Dalton R, Millogo N, Baldet T, et al. Larval development of the molecular forms of Anopheles gambiae (Diptera: Culicidae) in different habitats: a transplantation experiment. J Med Entomol. 2005;42:548-53.

51. Beier JC, Keating J, Githure Jl, Macdonald MB, Impoinvil DE, Novak RJ. Integrated vector management for malaria control. Malar J. 2008;7:S4.

52. Alphey L. Genetic control of mosquitoes. Annu Rev Entomol. 2014;59:205-24.

53. Burt A, Coulibaly M, Crisanti A, Diabate A, Kayondo JK. Gene drive to reduce malaria transmission in sub-Saharan Africa. J Responsible Innov. 2018:5:566-80.

54. Connolly JB, Mumford JD, Fuchs S, Turner G, Beech C, North AR, et al. Systematic identification of plausible pathways to potential harm via problem formulation for investigational releases of a population suppression gene drive to control the human malaria vector Anopheles gambiae in West Africa. Malar J. 2021;20:1.

55. Midega JT, Mbogo CM, Mwambi H, Wilson MD, Ojwang G, Mwangangi $\mathrm{JM}$, et al. Estimating dispersal and survival of Anopheles gambiae and Anopheles funestus along the Kenyan coast by using mark-release-recapture methods. J Med Entomol. 2007:44:923-9.

56. Takken W, Charlwood JD, Billingsley PF, Gort G. Dispersal and survival of Anopheles funestus and A. gambiae s.I. (Diptera: Culicidae) during the rainy season in southeast Tanzania. Bull Entomol Res. 2009;88:561-6.

57. Thomas CJ, Cross DE, Bogh C. Landscape movements of Anopheles gambiae malaria vector mosquitoes in rural Gambia. PLoS ONE. 2013;8:e68679.

58. Gillies MT. Studies on the dispersion and survival of Anopheles gambiae Giles in East Africa, by means of marking and release experiments. Bull Entomol Res. 1961;52:99-127.

\section{Publisher's Note}

Springer Nature remains neutral with regard to jurisdictional claims in published maps and institutional affiliations.
Ready to submit your research? Choose BMC and benefit from:

- fast, convenient online submission

- thorough peer review by experienced researchers in your field

- rapid publication on acceptance

- support for research data, including large and complex data types

- gold Open Access which fosters wider collaboration and increased citations

- maximum visibility for your research: over $100 \mathrm{M}$ website views per year

At BMC, research is always in progress.

Learn more biomedcentral.com/submissions 